\title{
Transcriptional profiling of adult Drosophila antennae by high-throughput sequencing
}

\author{
Meng-Shin Shiao ${ }^{1,2}$, Wen-Lang Fan ${ }^{1,2}$, Shu Fang ${ }^{1}$, Mei-Yeh Jade Lu' ${ }^{1}$ Rumi Kondo ${ }^{3}$ and Wen-Hsiung Li ${ }^{1,4^{*}}$
}

\begin{abstract}
Background: Antennae of fruit flies are the major organs responsible for detecting environmental volatiles, e.g., egg-laying substrates. An adult antenna contains many sensilla full of olfactory sensory neurons, where olfactory receptor (Or) genes are expressed. Each sensory neuron only expresses up to three receptors, making it difficult to estimate expression levels by conventional methods. In this study, we applied Illumina RNA sequencing (RNA-seq) to study the expression levels of Or and other genes in fly antennae.

Results: RNA from approximately 1,200 pairs of adult antennae from each sex of Drosophila melanogaster was used to obtain the antennal transcriptome of each sex. We detected approximately 12,000 genes expressed in antennae of either sex. The most highly expressed genes included pheromone-binding genes, transmembrane transporter genes, and sensory reception genes. Among the 61 annotated Or genes, we observed 53 and 54 genes (approximately 90\%) expressed (fragments per kilobase of exon per million fragments mapped (FPKM) > 0.05) in male and female antennae, respectively; approximately 25 genes were expressed with FPKM $>15$. Compared to previous studies, which extracted RNA from the whole body or head and used microarrays, antenna-specific transcriptomes obtained by RNA-seq provided more reliable estimates of gene expression levels and revealed many lowly expressed genes. Ninty-one genes, including one odorant-binding protein (Obp) gene and four Or genes, were differentially expressed between male and female antennae. These sexually biased genes were enriched on the X chromosome and showed enrichment in different gene ontology categories for male and female flies. The present and previous data together suggest that a gene family with putative immune response functions is related to pheromone detection and involved in the courtship behavior of male flies.
\end{abstract}

Conclusions: Tissue-specific RNA-seq is powerful for detecting lowly expressed genes. Our study provides new insight into the expression of olfactory-related genes in Drosophila antennae.

Keywords: Transcriptome; RNA-seq; Olfactory receptors; Odorant-binding proteins; Sexual bias

\section{Background}

Chemicals are detected by various categories of chemosensory receptors (CRs) of animals in order to find food and mates, define territories, and avoid danger. It was proposed that the numbers and complexities of CRs in animals evolved together with their living environments. For example, the house mouse Mus musculus possesses more than 1,500 functional genes encoding CRs in its genome, reflecting its adaptation to nocturnal life. In comparison, the number of functional CRs is significantly lower in

\footnotetext{
* Correspondence: whli@sinica.edu.tw

'Biodiversity Research Center, Academia Sinica, Taipei 11529, Taiwan ${ }^{4}$ Department of Ecology and Evolution, University of Chicago, Chicago, IL 60637, USA

Full list of author information is available at the end of the article
}

higher primates, likely due to their diurnal lifestyles or the emergence of a trichromatic color-vision system (Nei et al. 2008).

In insects, there are three major chemosensory gene families: odorant-binding protein $(\mathrm{Obp})$ genes, olfactory receptor (Or) genes, and gustatory $(\mathrm{Gr})$ genes. In the Drosophila melanogaster genome, approximately 60 genes each were annotated in the $\mathrm{Or}$ and $\mathrm{Gr}$ gene families, and approximately 50 genes were annotated in the $\mathrm{Obp}$ gene family. In general, these genes are responsible for sensing odors in the environment, such as from foods, egg-laying substrates, mates, and predators. Odorant and pheromone detection in insects is thought to contribute to species evolution. The European corn borer Ostrinia nubilalis consists of two races that use

\section{实}


two different 'blends' of sex pheromones. Females of one race only recognize pheromones secreted by males of the same race. This indicates that the female has evolved specific receptors to recognize race-specific pheromone blends. Such subtle differences may lead to reproductive isolation in the field between the two races, which could be the first step in speciation (Lassance et al. 2010). Drosophila sechellia, a Drosophila species solely found in the Seychelles archipelago, only utilizes one kind of fruit as its food resource and egg-laying substrate. Interestingly, the fruit is toxic to closely related Drosophila species, which diverged only approximately 500,000 (Drosophila simulans) to approximately $3 \times 10^{6}$ (D. melanogaster) years ago (Throckmorton 1975; Kliman et al. 2000). Previous studies found that the evolution of some chemosensory genes may have contributed to the specific host-plant selection in D. sechellia (Matsuo et al. 2007; Matsuo 2008).

In flies, olfactory sensory neurons are mainly distributed on sensilla of the adult antenna and maxillary palp (MP), and each neuron expresses only 1 to 3 Or genes (Vosshall et al. 1999; Yao et al. 2005). Gustatory sensory neurons are distributed on multiple body parts, including the proboscis, wing margins, legs, and ovipositor (Dunipace et al. 2001; Laissue and Vosshall 2008; Montell 2009). Olfactory receptors (ORs) detect environmental volatiles through 'smelling, while gustatory receptors (GRs) detect them by 'contact and tasting'. Both olfactory and gustatory organs express odorant-binding proteins (OBPs). Similar to the situation in vertebrates, hydrophobic odorant molecules are carried by OBPs through the lymph before they are released to ORs and GRs in sensory neurons. In flies, there are several hundred olfactory sensilla on an antenna, which is thus the most important 'nose' of Drosophila. Therefore, we focused on the transcriptional profiling of chemosensory genes on the antennae of both male and female flies ( $D$. melanogaster). Of course, it would also be interesting to see what other genes are expressed in the antennae and whether there are sexually differential expressions of genes in antennae.

One difficulty in studying chemosensory genes, particularly $O r$ and $G r$ genes, is that each gene is expressed by very few chemosensory neurons. For example, Drosophila sensilla have only about 1,200 neurons in total, and each Or gene is expressed in at most 20 neurons (Clyne et al. 1999; Vosshall et al. 1999; Dunipace et al. 2001). Therefore, a transcriptome from whole adult flies or heads might not represent expression patterns of $\mathrm{Or}$ and Gr genes or provide reliable data for statistical analyses because of low expressions of genes in a whole fly. Our previous study demonstrated that by conducting tissuespecific RNA-seq of the mouse olfactory epithelium, we obtained reliable estimates of OR gene expression levels (Shiao et al. 2012). In this study, we therefore used a next- generation sequencing platform to generate antennaspecific transcriptomes and studied expression patterns of chemosensory genes, mainly $\mathrm{Or}$ and $\mathrm{Obp}$ genes, in adult fly antennae.

\section{Methods}

Approximately 1,200 pairs of antennae from each sex of D. melanogaster Canton-S flies were collected for total RNA extraction. Only those flies that emerged within $1 \mathrm{~h}$ (usually in the morning) were collected for resection. Resected antennae were immediately preserved in approximately $100 \mu \mathrm{l}$ Trizol (kept on ice during resection) and stored at $-80^{\circ} \mathrm{C}$ before further processing.

The RNA isolation protocol we developed combined steps from conventional Trizol extraction and an RNeasy Micro Elute Kit (Qiagen, Venlo, Netherlands) with some modifications (described below) to obtain a maximum yield and quality of total RNA. We first spun down antennae in tubes at 13,000 rpm for $3 \mathrm{~min}$ at $4^{\circ} \mathrm{C}$, and then carefully combined antennae from all tubes into 1 MagNA Lyser Green Beads tube (Roche, Basel, Switzerland). The tissue was then disrupted in approximately $1 \mathrm{ml}$ Trizol with MagNA Lyser (Roche) at 7,000 rpm for $15 \mathrm{~s}$ each time for 4 or 5 times until the tissue was almost invisible. Between all 15-s disruptions, we cooled down the lysate on ice for $10 \mathrm{~s}$ to prevent RNA degradation. The tissue lysate was transferred from the Green Beads tube to a new RNase-free $1.5 \mathrm{ml}$ tube. Two hundred microliters of chloroform per microliter of tissue lysate was added to the lysate and well mixed by shaking vigorously for $15 \mathrm{~s}$ and was then allowed to sit at room temperature for 2 to $3 \mathrm{~min}$. The aqueous phase was separated by centrifuging the lysate at $13,000 \mathrm{rpm}$ at $4{ }^{\circ} \mathrm{C}$ for $15 \mathrm{~min}$ and then carefully transferring to a new $1.5 \mathrm{ml}$ tube. For RNA precipitation, we added $5 \mu \mathrm{l}$ Carrier RNA working solution (Qiagen) and $1 \mathrm{ml}$ of $70 \% \mathrm{EtOH}$ to the aqueous phase and mixed it well by carefully inverting the tubes. To obtain a greater amount of RNA, we kept the samples at $-80^{\circ} \mathrm{C}$ overnight to promote RNA precipitation. RNA isolation then followed the manufacture's protocol of the RNeasy Miro Elute Kit (Qiagen) with modifications of an increased volume $(700 \mu \mathrm{l})$ of RPE buffer and $80 \%$ ethanol to wash the RNA. Genomic DNA was removed by DNase I treatment for $15 \mathrm{~min}$ at room temperature. As there is only one major ribosomal RNA peak in Drosophila total RNA, the quality could not be evaluated by an Agilent Bioanalyzer. Instead, we evaluated the quality of total RNA by running 500 ng of total RNA on a $2 \%$ agarose gel.

For pair-end mRNA-seq library preparation, we used Illumina TrueSeq kits (San Diego, CA, USA). In total, $5 \mu \mathrm{g}$ of total RNA was used for mRNA enrichment by oligo-dT beads followed by cation-catalyzed fragmentation for 4 min at $94^{\circ} \mathrm{C}$. mRNA fragments were then converted into double-stranded cDNA by random priming followed by 
end repair. Fragmented cDNAs from two samples were then separately ligated to barcoded paired-end adaptors and subjected to 15 cycles of polymerase chain reaction (PCR) amplification followed by a sequencing reaction. For the data analysis, the 100-bp paired-end sequencing reads were mapped to the reference genome with TopHat 2.0.6 (Trapnell et al. 2009). We allowed two mismatches in each read when mapping to the reference genome. The expression level of a gene was estimated in terms of fragments per kilobase of exon per million fragments mapped (FPKM), and differentially expressed genes were identified using Cuffdiff 2.0.2 (Trapnell et al. 2012).

To confirm multiples of changes of sex-biased chemosensory genes, we conducted a quantitative reverse transcription (qRT)-PCR with NanoString technology. The probes of the target region of each gene are listed in Additional file 1: Table S1. For each gene, three technical duplicates were conducted with $100 \mathrm{ng}$ of total RNA from each sample, and actin $42 \mathrm{~A}($ Act $42 A)$ and ribosomal protein L32 (RpL32) were used as endogenous controls.

\section{Results and discussion Results}

We separately collected 2 total RNA samples from approximately 1,200 male flies and approximately $1,200 \mathrm{fe}-$ male virgin flies within $1 \mathrm{~h}$ after eclosion. The two samples were barcoded and paired-end (PE) sequenced in one Illumina Hiseq sequencing lane. Approximately $105 \times 10^{6}$ and $113 \times 10^{6} 100$-bp PE reads were respectively obtained from male and female flies. Overall, we detected approximately 12,000 (approximately $80 \%$ of approximately 15,000 genes annotated in the $D$. melanogaster genome) genes expressed in the antennae (Additional file 1: Table S2). It should be noted that genes were only considered to be expressed when their FPKM was $>0.05$; this criterion was chosen based on a suggestion in the previous literature (Toung et al. 2011). Among the 100 most highly expressed genes in male and female antennae, 92 genes overlapped between the 2 samples. These genes were enriched in the functional categories of pheromone/odorant binding, transmembrane transporter activity, and sensory perception (Table 1, Additional file 1: Tables S3 and S4). About $10 \%$ of the 100 most highly expressed genes were Obps, including lush $(\mathrm{Obp} 76 a)$, in agreement with a previous study (Anholt and Williams 2010) (Additional file 1: Table S4). lush was shown to respond to 11-cis-vaccenyl acetate and acts as a sex and aggregation pheromone in D. melanogaster (Kim et al. 1998; Xu et al. 2005; Ha and Smith 2006). However, we detected no significant difference in expression levels of lush between male and female flies. Five of the most highly expressed genes showed differential expression patterns between male and female antennae $(p<0.05$, with a false discovery rate (FDR) of $<0.05)$. These genes included four ribosomal genes (CR40677, CR41619,
CR34335, and 5.8SrRNA:CR40454) and one proteincoding gene with immune-induced function (immuneinduced molecule 3; IM3) (Additional file 1: Table S4).

\section{Or and Obp genes expressed in antennae}

We detected the expression of more than 50 Or genes, 40 $\mathrm{Obp}$ genes, and $35 \mathrm{Gr}$ genes in antennae of both male and female flies (with an FPKM of $>0.05$, Table 2, Additional file 1: Table S5). Among the chemosensory genes expressed, approximately $25 \mathrm{Or}$ and approximately $20 \mathrm{Obp}$ genes were expressed at moderately high expression levels (an FPKM of $>15$ ). For $\mathrm{Gr}$ genes, we detected fewer than five genes expressed at moderate or high levels, consistent with the view that most of the Gr genes are expressed in other body parts of flies. Kopp et al. (2008) used microarrays to study the transcriptome of Drosophila antennae, and observed that $51 \mathrm{Or}$ genes and $38 \mathrm{Obp}$ genes were expressed. They used flies at the same age as we did, i.e., $1 \mathrm{~h}$ after eclosion. We detected a similar number of expressed Or genes but more expressed $O b p$ genes by high-throughput sequencing. $O b p$ genes that were not detected by Kopp et al. were mostly lowly expressed (with an FPKM of $<15$ ).

Couto et al. (2005) identified 7 Or genes that were only expressed in the maxillary palp (MP) (MP-specific) and 11 Or genes that were only expressed in the larval stage (larva-specific). Among the seven MP-specific genes, six (Or33c, Or42a, Or46a, Or71a, Or85d, and Or85e) were expressed in our transcriptomes from adult antennae of at least one sex (with an FPKM of $>0.05$ ). However, most of the 6 genes were expressed at very low levels (at an FPKM of $<1$ ) except Or46a, which was expressed at FPKMs of 13.47 in males and 11.71 in females. We therefore concluded that the previously identified MP-specific expression pattern was due to the extremely low expression levels in antennae. Among the 11 larvaspecific genes identified by Couto et al. (2005), 5 (Or24a, Or30a, Or45a, Or59a, and Or94b) showed no expression in our transcriptomes (at an FPKM of $<0.05$ ). The other 6 genes (Or22c, Or33b, Or63a, Or74a, Or83a, and Or85c) showed very low expression levels in adult antennae (see Additional file 1: Table S5 for details).

Sensilla of flies can be classified into five distinct morphological classes: large basiconic, small basiconic, trichoid and ceoloconic (only on the antenna), and thin basiconic (on the antenna and maxillary palp) (Shanbhag et al. 2000). McBride and Arguello (2007) found that 4 Or genes (Or59b, Or85a, Or42b, and Or92a) and $2 \mathrm{Gr}$ genes (Gr21a and Gr63a) distributed on antennal basiconic sensilla 1 and 2 (ab1 and ab2) showed the most conserved pattern in adults. Interestingly, these six genes were expressed at a higher level than the average of all the $\mathrm{Or}$ and $\mathrm{Gr}$ genes (Additional file 1: Table S5), suggesting that they serve important functions in Drosophila antennae. 
Table 1 Enriched functional categories of genes highly expressed in both male and female antennae

\begin{tabular}{|c|c|c|c|c|}
\hline Category & $\begin{array}{l}\text { Enrichment } \\
\text { score }\end{array}$ & Term & $\begin{array}{c}\text { Number of } \\
\text { genes }\end{array}$ & $p$ value \\
\hline Cluster 1 & 9.79 & & & \\
\hline SMART & & SM00708:PhBP & 10 & 0.00 \\
\hline INTERPRO & & IPR006625:Insect pheromone-/odorant-binding protein PhBP & 10 & 0.00 \\
\hline INTERPRO & & IPR006170:Pheromone-/general odorant-binding protein, PBP/GOBP & 10 & 0.00 \\
\hline SP_PIR_KEYWORDS & & Disulfide bond & 10 & 0.00 \\
\hline UP_SEQ_FEATURE & & Disulfide bond & 10 & 0.01 \\
\hline
\end{tabular}

\section{Cluster 2}

GOTERM_MF_FAT

GOTERM_MF_FAT

GOTERM_MF_FAT

\section{Cluster 3}

GOTERM_BP_FAT

GOTERM_BP_FAT

GOTERM_BP_FAT

\section{Cluster 4}

GOTERM_CC_FAT

GOTERM_CC_FAT

GOTERM_CC_FAT

GOTERM_CC_FAT

GOTERM_CC_FAT

GOTERM_CC_FAT

\section{Cluster 5}

GOTERM_BP_FAT

GOTERM_BP_FAT

GOTERM_BP_FAT

\section{Cluster 6}

GOTERM_BP_FAT

GOTERM_MF_FAT

GOTERM_MF_FAT

GOTERM_MF_FAT

GOTERM_MF_FAT

GOTERM_CC_FAT

GOTERM_CC_FAT

8.86

$\begin{array}{lll}\text { GO:0015078 hydrogen ion transmembrane transporter activity } & 11 & 0.00 \\ \text { GO:0015077 monovalent inorganic cation transmembrane transporter activity } & 11 & 0.00 \\ \text { GO:0022890 inorganic cation transmembrane transporter activity } & 11 & 0.00\end{array}$

8.37

$\begin{array}{lll}\text { GO:0007606 sensory perception of chemical stimulus } & 14 & 0.00 \\ \text { GO:0007600 sensory perception } & 14 & 0.00 \\ \text { GO:0050890 cognition } & 14 & 0.00\end{array}$

7.42

$\begin{array}{lcr}\text { GO:0005743 mitochondrial inner membrane } & 15 & 0.00 \\ \text { GO:0019866 organelle inner membrane } & 15 & 0.00 \\ \text { GO:0031966 mitochondrial membrane } & 15 & 0.00 \\ \text { GO:0005740 mitochondrial envelope } & 15 & 0.00 \\ \text { GO:0031967 organelle envelope } & 15 & 0.00 \\ \text { GO:0031975 envelope } & 15 & 0.00\end{array}$

6.99

$\begin{array}{lll}\text { GO:0022904 respiratory electron transport chain } & 9 & 0.00 \\ \text { GO:0045333 cellular respiration } & 9 & 0.00 \\ \text { GO:0015980 energy derivation by oxidation of organic compounds } & 9 & 0.00\end{array}$

6.74

$\begin{array}{lll}\text { GO:0006123 mitochondrial electron transport, cytochrome c to oxygen } & 6 & 0.00 \\ \text { GO:0004129 cytochrome-c oxidase activity } & 6 & 0.00 \\ \text { GO:0015002 heme-copper terminal oxidase activity } & 6 & 0.00 \\ \text { GO:0016676 oxidoreductase activity, acting on heme group of donors, } & 6 & 0.00 \\ \text { oxygen as acceptor } & & \\ \text { GO:0016675 oxidoreductase activity, acting on heme group of donors } & 6 & 0.00 \\ \text { GO:0005751 mitochondrial respiratory chain complex IV } & 6 & 0.00 \\ \text { G0:0045277 respiratory chain complex IV } & 6 & 0.00\end{array}$

The enrichment score indicates the relative significance of a gene cluster among all clusters identified. Gene ontology (GO) terms with $p$ value of $<0.05$ and clusters with enrichment scores of $>5$ are shown. Please see Additional file 1: Table S3 for the full list of clusters with enrichment scores of $>1$.

Overall, Or genes were expressed at levels similar to genome-wide expression levels, while $\mathrm{Obp}$ genes showed a wider range of expression levels (Figure 1). Or83b
(Orco) showed an extraordinarily high expression level (FPKMs of 1219.24 and 1273.13 in male and female antennae, respectively) among Or genes, in agreement with 
the view that $O r 83 b$ is a widely expressed receptor in all neurons (Laissue and Vosshall 2008). Among the three chemosensory gene families, we only observed five genes with significantly different expression levels between male and female flies $(p<0.05$, with an FDR of $<0.05$, Table 3). This indicates that male and female flies do not show much difference in detecting environmental volatiles.

\section{Sex-biased chemosensory genes in adult antennae}

Among the 91 sexually dimorphic genes, we only observed five chemosensory genes that showed differential expression (Table 3, Additional file 1: Table S6). Or47b and $0 r 67 d$ showed higher expression levels in male flies, while Or59b, Or9a, and Obp19b showed higher expression levels in female flies. Or $47 b$ and Or67d were proposed as being involved in sociosexual interactions in flies, such as courtship and mating behaviors (Lone and Sharma 2012). In particular, the ablation of $0 r 47 b$ neurons had the most dramatic effect on the sociosexual interaction behaviors (Lone and Sharma 2012).

Using microarrays, Kopp et al. (2008) found 406 differentially expressed genes between male and female antennae among 9,830 genes examined, including 20 Or and Obp genes (with a FDR of <0.2). Among the $20 \mathrm{Or}$ and Obp genes, 14 Or genes (approximately 23\%) and $6 \mathrm{Obp}$ genes (approximately 12\%) showed greater proportions of sex-biased patterns compared to the entire transcriptome (approximately 4\%). Although we observed a much smaller number of genes (91 genes) that are sexually biased, chemosensory genes still showed a higher proportion $(5 \%)$ compared to the entire transcriptome $(0.7 \%)$ (Fisher's exact test, $p<0.0001$ ).

If we used less-stringent criteria ( $p<0.05$ without considering the FDR) for determining the sexual bias of chemosensory genes, we observed 18 chemosensory genes and 455 genes from the entire transcriptome that showed differential expressions between male and female flies. Among the 18 genes, 8 genes were also observed by Kopp et al. (Table 4). On the other hand, based on more stringent criteria, four of the five sexual dimorphic chemosensory genes identified in our study were also identified by Kopp et al.; the only exception was Or59b. We further confirmed differences in expression levels between male

Table 2 Numbers of genes in three chemosensory gene families

\begin{tabular}{|c|c|c|c|c|c|c|}
\hline & \multicolumn{2}{|r|}{ Or } & \multicolumn{2}{|c|}{$O b p$} & \multicolumn{2}{|c|}{$G r$} \\
\hline & Male & Female & Male & Female & Male & Female \\
\hline Annotated & \multicolumn{2}{|c|}{61} & \multicolumn{2}{|c|}{50} & \multicolumn{2}{|c|}{63} \\
\hline FPKM > 0.05 & 53 & 54 & 44 & 43 & 34 & 37 \\
\hline FPKM > 15 & 23 & 26 & 19 & 21 & 2 & 4 \\
\hline
\end{tabular}

Please see Additional file 1: Table S5 for the complete gene list. Or, olfactory receptor; Obp, odorant-binding protein; Gr, gustatory receptor. and female samples for sex-biased chemosensory genes using the NanoString qRT-PCR technology. Expression fold changes between two samples from qRT-PCR are well corresponded to the sequencing results (Table 4).

\section{Enrichment of sex-biased genes on the $X$ chromosome}

In total, 91 genes showed differential expression levels between male and female flies $(p<0.05$, with an FDR of $<0.05$, Figure 2). Interestingly, 45 of the 91 genes were located on the X chromosome (among a total of 1,956 genes expressed on the $\mathrm{X}$ chromosome), while only 35 genes were found on autosomes (out of a total of 10,118 genes expressed on autosomes); 11 sex-biased genes on unidentified contigs were not included (Additional file 1 : Table S7). When we used a less-stringent criterion $(p<$ 0.05 without considering the FDR), 185 genes showed a sexual bias on the $\mathrm{X}$ chromosome and 241 genes on autosomes (after excluding genes on the unidentified contigs). Both analyses suggested that sex-biased genes are highly enriched on the X chromosome $(p<0.0001)$. Note that we did not consider genes on unassigned contigs (Additional file 1: Table S7).

As mentioned above, among the $45 \mathrm{X}$-linked genes that showed sexual bias, 18 were more highly expressed in males (male-biased), and 27 genes were more highly expressed in females (female-biased) with the more stringent criteria. With the less-stringent criteria, $125 \mathrm{X}$-linked genes were male-biased and 116 genes were femalebiased. We found no enrichment of female-biased genes on the $\mathrm{X}$ chromosome. It was proposed that sex-biased genes are not randomly distributed across the Drosophila genome (Parisi et al. 2003; Sturgill et al. 2007). In particular, $\mathrm{X}$ chromosomes are underrepresented in male-biased genes but are possibly enriched in femalebiased genes (feminized). However, the transcriptome of antennae revealed that both male- and female-biased genes were enriched on the X chromosome of D. melanogaster (Figure 3A, Additional file 1: Table S7). Moreover, expression levels $\left(\log _{2}\right.$ (FPKM)) of male-biased genes tended to be higher than those of female-biased genes on the $\mathrm{X}$ chromosome (Figure 3B). This suggests that the $\mathrm{X}$ chromosome does not show 'demasculinization' in adult antennae.

The gene enrichment analyses (performed on DAVID; Jiao et al. 2012) showed that genes involved in dosage compensation, protein-DNA complex assembly, and immunity more often showed higher expression levels in males than females. Good examples are the two RoX genes, which are non-coding RNA genes and were proposed to be involved in dosage compensation of the $\mathrm{X}$ chromosome in male flies (see review by Ilik and Akhtar 2009). On the other hand, genes involved in chitin binding, sexual differentiation, and signaling more often 


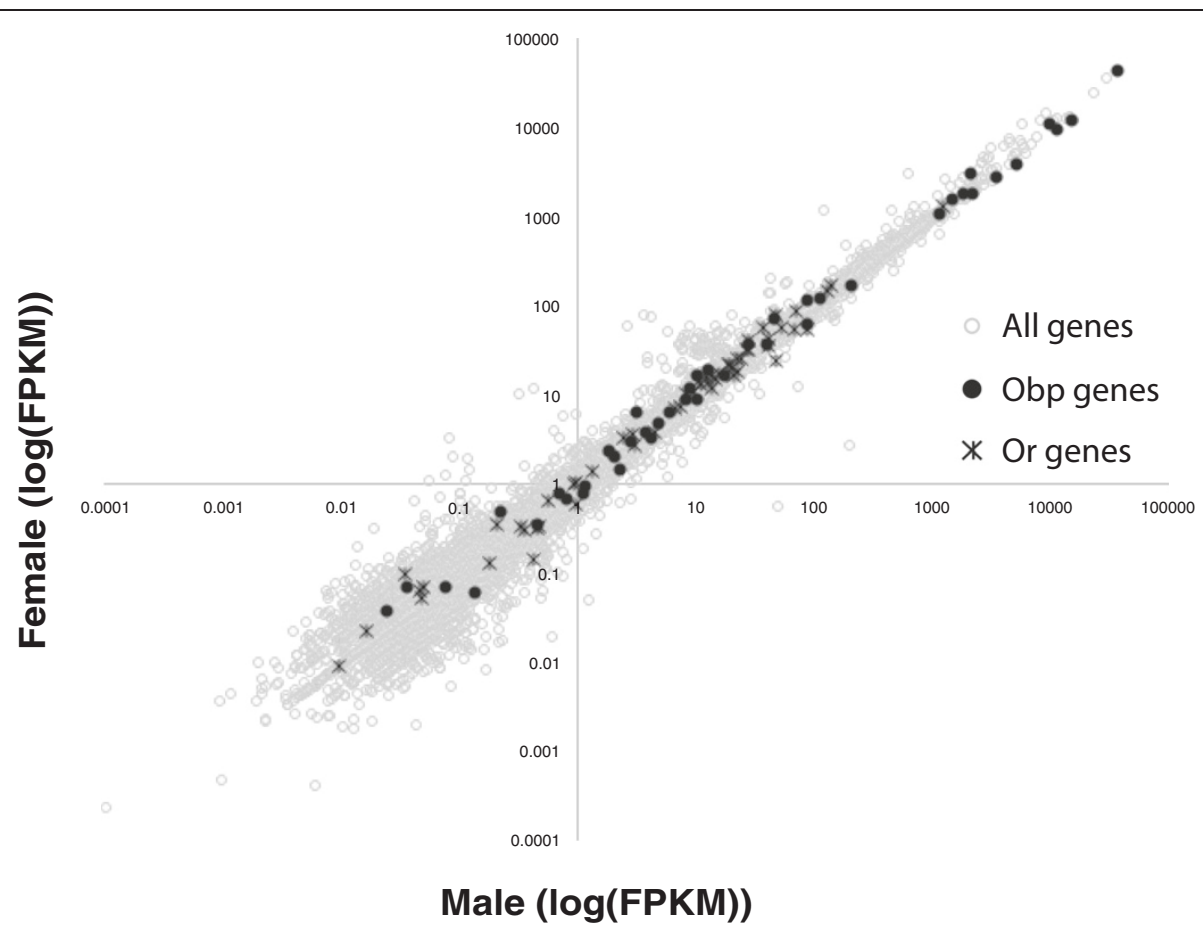

Figure 1 Correlation of expression levels in antennae between male and female flies. Black dots, Obp genes; stars, Or genes; open circles, all other genes.

showed higher expression levels in females than males, e.g., yolk protein 1, 2, and $3(Y p 1, Y p 2$, and $Y p 3)$ genes.

\section{Possible involvement of immune-induced genes in pheromone detection}

A previous study found that when male flies of D. melanogaster encountered female flies from the closely related species, $D$. simulans, their courtship behavior significantly dropped within 5 min (Ellis and Carney 2009). In addition, expression levels of nine genes were significantly altered, mostly down-regulated, in males. Interestingly, eight of the nine genes have putative function in immune responses including immune-induced molecule 1, 10, and 23 (IM1, IM10 and IM23) genes and 2 novel genes (CG15065 and CG16836). Three of the IM genes (IM3, IM4, and IM14) had the most abundant expression levels in our transcriptomes. We conducted protein blast searches and found that CG15065 and CG16836 have the same immune- response protein domains as the other IM genes and formed a tight cluster with $I M 1, I M 2$, and $I M 3$ (i.e., within $5 \mathrm{~kb})$ on chromosome 2R. However, mechanisms of immune-response genes involved in male courtship behavior are still unknown. From our transcriptomes, we found that all seven IM genes and two novel genes showed male-biased expression patterns to a certain degree (Table 5). That is, this gene family has a higher expression level in antennae of male flies than in antennae of female flies, although some of them were not statistically significant. Therefore, we propose that the IM gene family is involved in pheromone reception in antennae, particularly in male flies.

\section{Discussion}

With a stringent criterion, we identified only five sexbiased $O r$ and $O b p$ genes in adult antennae. However, with a less-stringent criterion, we identified 18 chemosensory

Table 3 Chemosensory genes differentially expressed between male and female flies

\begin{tabular}{|c|c|c|c|c|c|c|c|}
\hline Gene ID & Gene name & Chromosome & Male (FPKM) & Female (FPKM) & Fold change (M/F) & $p$ & FDR \\
\hline FBgn0031110 & Obp19b & $x$ & 46.05 & 70.89 & 0.65 & $* * *$ & 0.04 \\
\hline FBgn0026385 & Or47b & $2 R$ & 88.46 & 52.12 & 1.70 & $* * *$ & 0.00 \\
\hline FBgn0034865 & Or59b & $2 R$ & 48.20 & 76.20 & 0.63 & $* * *$ & 0.02 \\
\hline FBgn0036080 & Or67d & $3 \mathrm{~L}$ & 46.65 & 23.90 & 1.95 & $* * *$ & 0.00 \\
\hline FBgn0030204 & Or9a & $x$ & 36.24 & 54.41 & 0.67 & $* * *$ & 0.04 \\
\hline
\end{tabular}

*** $p<0.001$. 
Table 4 Sex-biased chemosensory genes

\begin{tabular}{|c|c|c|c|c|c|c|c|c|c|}
\hline & \multirow[t]{2}{*}{ Gene name } & \multirow[t]{2}{*}{ Gene ID } & \multicolumn{6}{|c|}{ Shiao et al. } & \multirow{2}{*}{$\begin{array}{c}\text { Kopp et al. } \\
\text { Expression leve }\end{array}$} \\
\hline & & & Male (FPKM) & Female (FPKM) & $\mathrm{FC}(\mathrm{F} / \mathrm{M})$ & $p$ & FDR & qRT-PCR (FC) & \\
\hline \multirow[t]{10}{*}{ Shiao et al. only } & Gr2a & FBgn0027796 & 0.63 & 0.20 & 0.32 & 0.01 & 0.58 & $\mathrm{NA}^{1}$ & $N A^{2}$ \\
\hline & Obp19c & FBgn0031111 & 12.57 & 18.45 & 1.47 & 0.01 & 0.55 & 1.45 & Low \\
\hline & Obp44a & FBgn0033268 & 85.73 & 115.43 & 1.35 & 0.03 & 1.00 & 1.24 & High \\
\hline & Obp49a & FBgn0050052 & 27.64 & 36.45 & 1.32 & 0.03 & 1.00 & 1.37 & High \\
\hline & Obp56e & FBgn0034471 & 10.30 & 16.05 & 1.56 & 0.03 & 1.00 & 1.77 & Medium \\
\hline & Obp57a & FBgn0043535 & 87.76 & 60.06 & 0.68 & 0.00 & 0.17 & 0.69 & High \\
\hline & Obp99b & FBgn0039685 & 3.18 & 6.17 & 1.94 & 0.02 & 0.77 & 1.50 & Low \\
\hline & Or59b & FBgn0034865 & 48.20 & 76.20 & 1.58 & 0.00 & 0.02 & 1.40 & Medium \\
\hline & Or65b & FBgn0041624 & 66.81 & 51.96 & 0.78 & 0.02 & 0.82 & 0.74 & Low \\
\hline & Or98b & FBgn0039582 & 0.43 & 0.14 & 0.34 & 0.03 & 1.00 & 0.88 & $N A^{2}$ \\
\hline \multirow[t]{8}{*}{ Overlap } & Gr21a & FBgn0041250 & 13.46 & 17.85 & 1.33 & 0.03 & 1.00 & 1.31 & High \\
\hline & Obp19a & FBgn0031109 & 2093.55 & 2986.89 & 1.43 & 0.04 & 1.00 & 1.42 & High \\
\hline & Obp19b & FBgn0031110 & 46.05 & 70.89 & 1.54 & 0.00 & 0.04 & 1.29 & High \\
\hline & Obp22a & FBgn0043539 & 0.18 & 0.00 & 0.00 & 0.05 & 1.00 & $N A^{1}$ & Low \\
\hline & Or47b & FBgn0026385 & 88.46 & 52.12 & 0.59 & 0.00 & 0.00 & 0.57 & High \\
\hline & Or67d & FBgn0036080 & 46.65 & 23.90 & 0.51 & 0.00 & 0.00 & 0.51 & High \\
\hline & Or7a & FBgn0030016 & 27.98 & 39.62 & 1.42 & 0.00 & 0.23 & 1.32 & Medium \\
\hline & Orga & FBgn0030204 & 36.24 & 54.41 & 1.50 & 0.00 & 0.04 & 1.45 & Medium \\
\hline \multirow[t]{12}{*}{ Kopp et al. only } & Gr64f & FBgn0052255 & 11.93 & 16.33 & 1.37 & 0.14 & 1.00 & 1.26 & Medium \\
\hline & Obp69a & FBgn0011279 & 5132.32 & 3815.70 & 0.74 & 0.12 & 1.00 & 0.69 & High \\
\hline & Obp84a & FBgn0011282 & 1832.46 & 1764.42 & 0.96 & 0.81 & 1.00 & 0.93 & High \\
\hline & Or13a & FBgn0030715 & 13.62 & 16.36 & 1.20 & 0.21 & 1.00 & 1.22 & Low \\
\hline & Or22b & FBgn0026397 & 71.33 & 84.25 & 1.18 & 0.11 & 1.00 & 1.16 & Medium \\
\hline & Or2a & FBgn0023523 & 8.33 & 9.94 & 1.19 & 0.29 & 1.00 & 0.85 & Low \\
\hline & Or35a & FBgn0028946 & 19.73 & 21.75 & 1.10 & 0.47 & 1.00 & 1.14 & High \\
\hline & Or42b & FBgn0033043 & 140.30 & 168.79 & 1.20 & 0.07 & 1.00 & 1.19 & High \\
\hline & Or82a & FBgn0041621 & 9.44 & 10.55 & 1.12 & 0.51 & 1.00 & 1.10 & Medium \\
\hline & Or85a & FBgn0037576 & 10.55 & 13.78 & 1.31 & 0.17 & 1.00 & 1.16 & Medium \\
\hline & Or85c & FBgn0037591 & 2.92 & 3.64 & 1.25 & 0.37 & 1.00 & 0.82 & Low \\
\hline & Or92a & FBgn0038798 & 127.15 & 144.45 & 1.14 & 0.21 & 1.00 & 1.08 & Medium \\
\hline
\end{tabular}

Sex-biased genes identified in this study (Shiao et al. 2012) and in Kopp et al. (2008) and overlap between two studies. FC, fold changes of fragments per kilobase of exon per million fragments mapped (FPKM) values and qRT-PCR results between females (F) and males (M); FDR, false discovery rate; NA ${ }^{1}$, fold change was too small based on sequencing results, and we therefore did not design probes for the qRT-PCR analysis; NA ${ }^{2}$, not detected by Kopp et al.

genes with a sex-biased expression pattern, including eight genes from a previous study (Kopp et al. 2008). There are three possible reasons for the different results between our study and those of Kopp et al. (2008). First, drawbacks of the array-based approach are a reliance upon existing knowledge of genome sequences, high background levels owing to cross-hybridization, and a limited dynamic range of detection owing to both the background and saturation of signals. Second, genes showing sexual dimorphism in Kopp et al. were determined by both interspecific or intraspecific factors. Kopp et al. performed an analysis of variance (ANOVA) test by considering three factors: sex, species, and the sex-species interaction; genes that showed significant sex or sex-species interaction were then considered sexually dimorphic. However, we only considered 'sex' in our study. Therefore, it is expected that more genes were determined as sexually dimorphic by Kopp et al. Third, it was observed that although array-based and sequencing-based approaches agree fairly well for genes with medium levels of expression, correlations were found to be very low for genes with either low or high expression levels (Wang et al. 2009). 


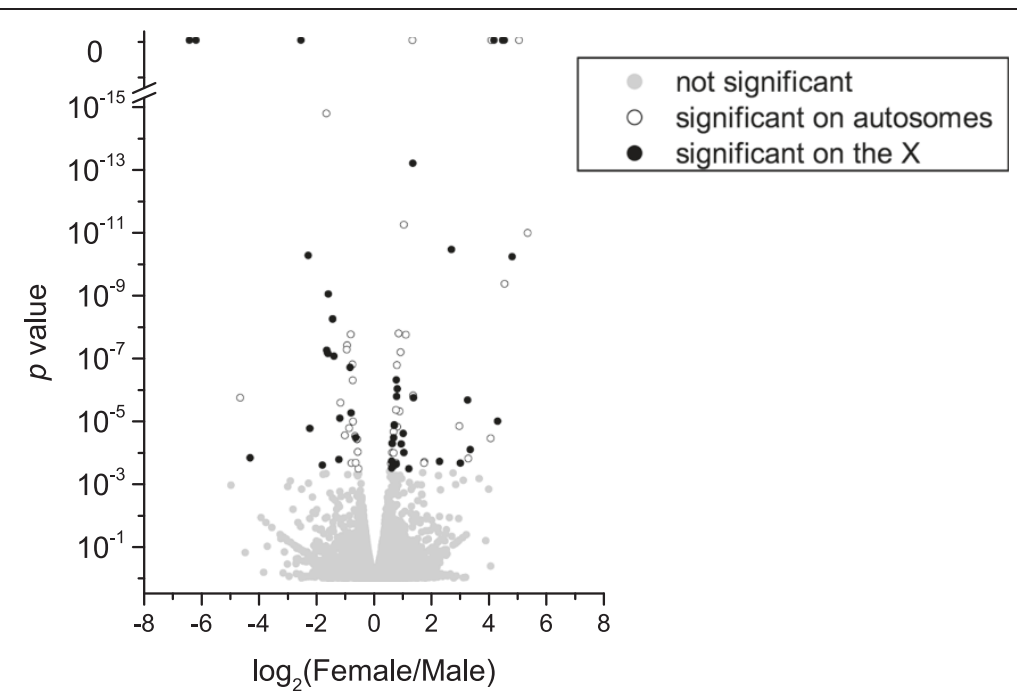

Figure 2 Volcano plot demonstrating significance of female-to-male expression ratio (multiples of change) for each gene. Open circles indicate autosomal genes showing different expression levels between male and female flies; black dots indicate X-linked genes showing different expression levels between males and females (45 genes); gray dots indicate genes showing no significant difference in expression levels.

Most of the published papers quantified the expression of chemosensory genes in antennae by obtaining total RNA from adult fly heads. The reason was likely because it is difficult to collect sufficient amounts of RNA from antennae. However, by comparing expression levels of $\mathrm{Obp}$ and $\mathrm{Or}$ genes in adult heads and antennae, we concluded that expression profiles of chemosensory genes in the adult head do not reflect expression profiles in antennae (Figure 4). Expression levels of genes in the adult head were retrieved from the FlyBase FlyAtlas database. In order to compare expression levels of genes between the FlyAtlas database and our data, we normalized expression levels of genes with the expression levels of
Act $42 A$ (for $\mathrm{Or}$ genes) and $\mathrm{Act} 5 \mathrm{C}$ (for $\mathrm{Obp}$ genes) genes in male antennae obtained in this study. For example, we obtained the normalized expression level of Or83b of the adult head using the formula: $\left(\mathrm{FPKM}_{\mathrm{O} 83} \mathrm{~B}\right.$ in adult head $\left./ \mathrm{FPKM}_{\text {Act } 42 A \text { in adult head }}\right) \times \mathrm{FPKM}_{\text {Act } 42 A}$ in male antennae. We used different genes to normalize expression levels of $\mathrm{Or}$ and $\mathrm{Obp}$ genes in view of the abundance differences in expression levels of the two gene families.

By comparing expression profiles of $\mathrm{Obp}$ genes in the antennae and head (Figure 4A), we concluded that the two profiles differed. Previously, Anholt et al. (2003) suggested that Obp99a and Obp99b were more highly expressed in female and male heads, respectively. In our
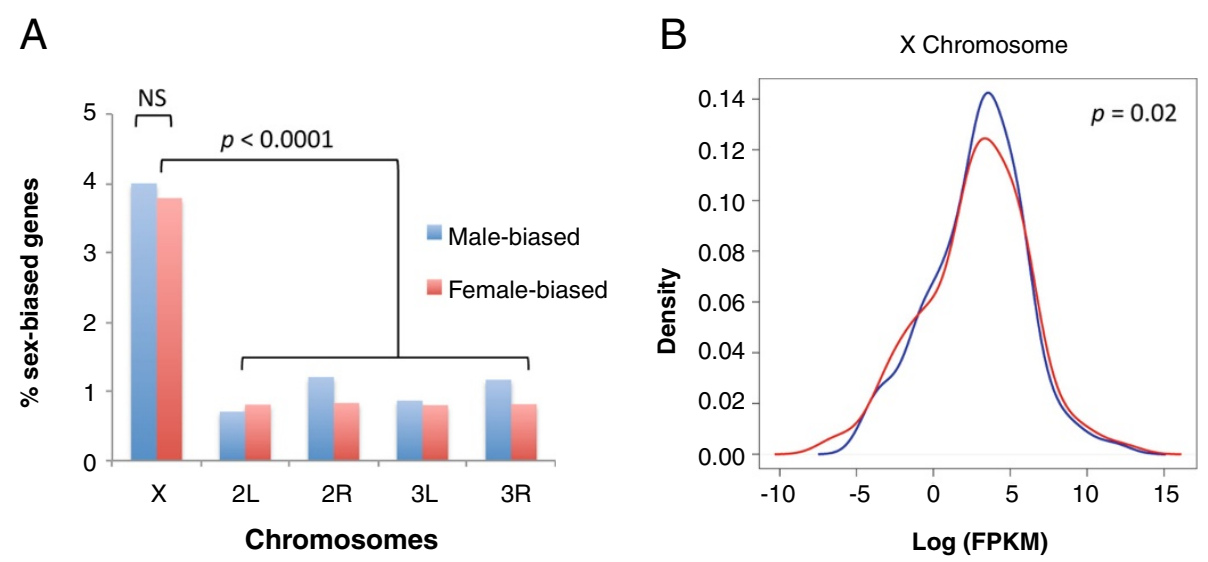

Figure 3 Proportion (A) and density plot (B) of expression levels of sex-biased genes on each chromosome. (A) Proportion of sex-biased genes (number of sex-biased genes/total expressed genes on each chromosome). Blue and red bars respectively show proportions of male- and female-biased genes on each chromosome. Please see Additional file 1: Table S7 for detailed data. Chromosome 4 is not shown due to the small gene numbers. (B) Density plot of expression levels of sex-biased genes on the X chromosome in antennae. Male-biased genes are shown in blue and female-biased genes in red. Significance was assessed by a chi-squared test. 
Table 5 Immune response (IM) genes expressed in antennae

\begin{tabular}{lccccrrr}
\hline Gene ID & Gene name & \multicolumn{1}{c}{ Locus } & Male (FPKM) & Female (FPKM) & Fold change (M/F) & $\boldsymbol{p}$ & FDR \\
\hline FBgn0034329 & IM1 & 2R:14271456-14271883 & 158.20 & 85.47 & -0.89 & 0.00 & 0.00 \\
FBgn0025583 & IM2 & 2R:14274101-14274535 & 456.40 & 303.76 & -0.59 & 0.00 & 0.02 \\
FBgn0040736 & IM3 & 2R:14275624-14275995 & 1129.01 & 635.11 & -0.83 & 0.00 & 0.00 \\
FBgn0040653 & IM4 & 2R:16756340-16756826 & 1504.47 & $1,060.94$ & -0.50 & 0.00 & 0.14 \\
FBgn0033835 & IM10 & 2R:9294510-9297458 & 51.77 & 43.54 & -0.25 & 0.16 & 1.00 \\
FBgn0067905 & IM14 & 2R:16757896-16758183 & 3256.23 & $2,453.80$ & -0.41 & 0.01 & 0.35 \\
FBgn0034328 & IM23 & 2R:14270208-14270737 & 6.26 & 2.43 & -1.37 & 0.00 & 0.24 \\
FBgn0040734 & CG15065 & 2R:14277462-14277752 & 141.29 & 78.45 & -0.85 & 0.02 & 0.79 \\
FBgn0040735 & CG16836 & 2R:14276599-14276963 & 113.38 & 72.79 & -0.64 & 0.01 & 0.47 \\
\hline
\end{tabular}

transcriptomes, however, only Obp99b was more highly expressed in female antennae (Table 4). This suggests that $\mathrm{Obp}$ genes may have different functions in the adult head than in antennae, in agreement with previous findings that $\mathrm{Obp}$ genes are also expressed in other tissues, such as the fat body and male accessory gland.

Expression levels of $\mathrm{Or}$ genes in the adult head were extremely low based on the FlyAtlas database (Figure 4B). This might have been due to data on FlyAtlas not being obtained from antennae and our transcriptomes of antennae showed much higher expression levels.

\section{Conclusion}

In this study, we demonstrated that RNA-seq provided a powerful tool in detecting genes with very low expression levels. With the latest technology, we detected expression of most of the annotated Or and Obp genes. We also observed several sex-biased chemosensory genes and showed that sex-biased genes are enriched on the $\mathrm{X}$ chromosome. We also showed that immuneinduced genes might be involved in the pheromone detection. The results of this study provide new insight into the expression of olfactory-related genes in the antennae of fruit flies.

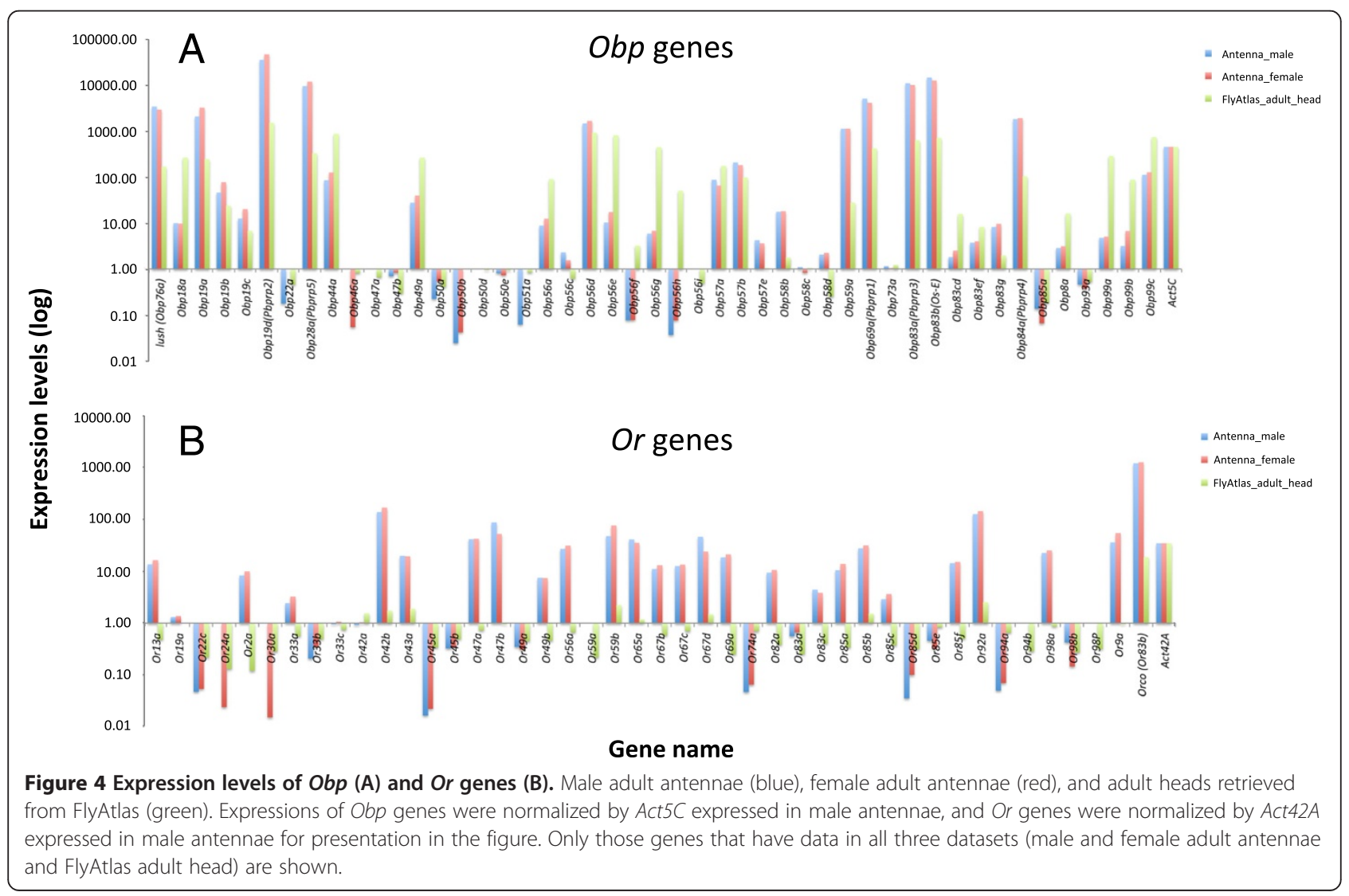




\section{Additional file}

Additional file 1: Probes targeted regions, expression levels of genes, functional clustering, highly expressed genes, and sexually biased genes. Table S1 Probes targeted regions in each gene for qRTPCR based on NanoString technology. Table S2 Expression levels of genes in antennae. FDR: faulse discovery rate. Table S3 Functional clustering of the most expressed genes in male and female antennae. Ninety two genes overlap between two samples. Stringency level: High, $p>0.05$, enrichment score $>1$. Table S4 The 100 most highly expressed genes in male and female antennae. Those belong to the 100 most highly expressed genes in male only are marked in blue while those belong to the 100 most highly expressed genes in female only are marked in red. Table S5 Expression levels of Gr genes, Or genes and Obp genes in adult antennae. 'MP only' indicates that the genes expressed in maxillary pulp only based on Couto et al. (2005). 'L' indicates that the genes expressed in both larvae and adult antennae while 'L only' indicates that the genes expressed exclusively in larvae but not in adult antennae (Couto et al. 2005). 'Most conserved' genes were identified by McBride and Arguello (2007). Table S6 Genes expressed differentially between male and female antennae (sex-biased) based on a more stringent criterion $(p<0.05$ and FDR < 0.05). Table S7 Number of sexually biased genes on each chromosome. Percentage of biased genes were calculated by the number of biased genes divided by the expressed genes on each chromosome and shown in the parentheses.

\section{Competing interests}

The authors declare that they have no competing interests.

\section{Authors' contributions}

MSS carried out the dissection and performed the analysis. MSS, RK, and WHL drafted the manuscript, and participated in the design of the study and coordination. WLF performed the analysis of RNA-seq. SF provided fly samples and participated in experiment designs. MYJL performed RNA-seq. All authors read and approved the final manuscript.

\section{Acknowledgements}

This study was supported by a grant the National Research Council, Taiwan (NSC99-2321-B-001-041-MY2). MS Shiao was supported by a postdoctoral fellowship from Academia Sinica, Taiwan. Special thanks go to CT Ting and Gene $\mathrm{Ng}$ for inspiring discussions.

\section{Author details}

${ }^{1}$ Biodiversity Research Center, Academia Sinica, Taipei 11529, Taiwan. ${ }^{2}$ Genomics Research Center, Academia Sinica, Taipei 11529, Taiwan.

${ }^{3}$ Graduate School of Humanities and Sciences, Ochanomizu University, Tokyo 112-8610, Japan. ${ }^{4}$ Department of Ecology and Evolution, University of Chicago, Chicago, IL 60637, USA.

\section{Received: 3 May 2013 Accepted: 15 July 2013}

Published: 13 November 2013

\section{References}

Anholt RR, Williams TI (2010) The soluble proteome of the Drosophila antenna. Chem Senses 35:21-30

Anholt RR, Dilda CL, Chang S, Fanara JJ, Kulkarni NH, Ganguly I, Rollmann SM, Kamdar KP, Mackay TF (2003) The genetic architecture of odor-guided behavior in Drosophila: epistasis and the transcriptome. Nat Genet 35:180-184

Clyne PJ, Warr CG, Freeman MR, Lessing D, Kim J, Carlson JR (1999) A novel family of divergent seven-transmembrane proteins: candidate odorant receptors in Drosophila. Neuron 22:327-338

Couto A, Alenius M, Dickson BJ (2005) Molecular, anatomical, and functional organization of the Drosophila olfactory system. Curr Biol 15:1535-1547

Dunipace L, Meister S, McNealy C, Amrein H (2001) Spatially restricted expression of candidate taste receptors in the Drosophila gustatory system. Curr Biol 11:822-835

Ellis LL, Carney GE (2009) Drosophila melanogaster males respond differently at the behavioural and genome-wide levels to Drosophila melanogaster and Drosophila simulans females. J Evol Biol 22:2183-2191
Ha TS, Smith DP (2006) A pheromone receptor mediates 11-cis-vaccenyl acetateinduced responses in Drosophila. J Neurosci 26:8727-8733

llik I, Akhtar A (2009) Rox RNAs: non-coding regulators of the male X chromosome in flies. RNA Biol 6:113-121

Jiao X, Sherman BT, Huang DW, Stephens R, Baseler MW, Lane HC, Lempicki RA (2012) David-WS: a stateful web service to facilitate gene/protein list analysis. Bioinformatics 28:1805-1806

Kim MS, Repp A, Smith DP (1998) Lush odorant-binding protein mediates chemosensory responses to alcohols in Drosophila melanogaster. Genetics 150:711-721

Kliman RM, Andolfatto P, Coyne JA, Depaulis F, Kreitman M, Berry AJ, McCarter J, Wakeley J, Hey J (2000) The population genetics of the origin and divergence of the Drosophila simulans complex species. Genetics 156:1913-1931

Kopp A, Barmina O, Hamilton AM, Higgins L, Mclntyre LM, Jones CD (2008) Evolution of gene expression in the Drosophila olfactory system. Mol Biol Evol 25:1081-1092

Laissue PP, Vosshall LB (2008) The olfactory sensory map in Drosophila. Adv Exp Med Biol 628:102-114

Lassance JM, Groot AT, Lienard MA, Antony B, Borgwardt C, Andersson F, Hedenström E, Heckel DG, Löfstedt C (2010) Allelic variation in a fatty-acyl reductase gene causes divergence in moth sex pheromones. Nature 466:486-489

Lone SR, Sharma VK (2012) Or47b receptor neurons mediate sociosexual interactions in the fruit fly Drosophila melanogaster. J Biol Rhythms 27:107-116

Matsuo T (2008) Genes for host-plant selection in Drosophila. J Neurogen 22:195-210

Matsuo T, Sugaya S, Yasukawa J, Aigaki T, Fuyama Y (2007) Odorant-binding proteins OBP57D and OBP57E affect taste perception and host-plant preference in Drosophila sechellia. PLoS Biol 5:e118

McBride CS, Arguello JR (2007) Five Drosophila genomes reveal nonneutral evolution and the signature of host specialization in the chemoreceptor superfamily. Genetics 177:1395-1416

Montell C (2009) A taste of the Drosophila gustatory receptors. Curr Opin Neurobiol 19:345-353

Nei M, Niimura Y, Nozawa M (2008) The evolution of animal chemosensory receptor gene repertoires: roles of chance and necessity. Nat Rev Genet 9:951-963

Parisi M, Nuttall R, Naiman D, Bouffard G, Malley J, Andrews J, Eastman S, Oliver B (2003) Paucity of genes on the Drosophila X chromosome showing malebiased expression. Science 299:697-700

Shanbhag SR, Muller B, Steinbrecht RA (2000) Atlas of olfactory organs of Drosophila melanogaster 2. Internal organization and cellular architecture of olfactory sensilla. Arthrop Struct Dev 29:211-229

Shiao MS, Chang AY, Liao BY, Ching YH, Lu MY, Chen SM, Li WH (2012) Transcriptomes of mouse olfactory epithelium reveal sexual differences in odorant detection. Genome Biol Evol 4:703-712

Sturgill D, Zhang Y, Parisi M, Oliver B (2007) Demasculinization of X chromosomes in the Drosophila genus. Nature 450:238-241

Throckmorton LH (1975) The phylogeny, ecology and geography of Drosophila. Plenum Publishing, New York

Toung JM, Morley M, Li M, Cheung VG (2011) RNA-sequence analysis of human B-cells. Genome Res 21:991-998

Trapnell C, Pachter L, Salzberg SL (2009) TopHat: discovering splice junctions with RNA-seq. Bioinformatics 25:1105-1111

Trapnell C, Hendrickson DG, Sauvageau M, Goff L, Rinn JL, Pachter L (2012) Differential analysis of gene regulation at transcript resolution with RNA-seq. Nat Biotechnol 31:46-53

Vosshall LB, Amrein H, Morozov PS, Rzhetsky A, Axel R (1999) A spatial map of olfactory receptor expression in the Drosophila antenna. Cell 96:725-736

Wang Z, Gerstein M, Snyder M (2009) RNA-seq: a revolutionary tool for transcriptomics. Nat Rev Genet 10:57-63

Xu P, Atkinson R, Jones DN, Smith DP (2005) Drosophila OBP LUSH is required for activity of pheromone-sensitive neurons. Neuron 45:193-200

Yao CA, Ignell R, Carlson JR (2005) Chemosensory coding by neurons in the coeloconic sensilla of the Drosophila antenna. J Neurosci 25:8359-8367

\section{doi:10.1186/1810-522X-52-42}

Cite this article as: Shiao et al:: Transcriptional profiling of adult Drosophila antennae by high-throughput sequencing. Zoological Studies 2013 52:42 